\title{
Review: repetitive transcranial magnetic stimulation has Additional intormation antidepressant effects
}

appears on the

Evidence-Based

Mental Health

website

www.ebmentalhealth. com

McNamara B, Ray JL, Arthurs OJ, et al. Transcranial magnetic stimulation for depression and other psychiatric disorders. Psychol Med 2001 Oct;31:1141-46.

\section{QUESTION: In patients with mood disorders or schizophrenia, how effective is repetitive transcranial magnetic stimulation (rTMS)?}

\section{Data sources}

Studies were identified by searching Medline (1966 to Jan 2000), EMBASE/Excerpta Medica (1980 to Jan 2000), Biological Abstracts, Index of Scientific and Technical Proceedings, the Meta Register of Controlled Trials, the national register, the Cochrane Library, and 5 TMS related internet sites.

\section{Study selection}

Studies were selected if they were randomised, placebo controlled trials of rTMS.

For correspondence Dr $S$ Boniface,

Department of Clinical

Neurophysiology,

Addenbrooke's

Hospital, Cambridge,

UK.sjb80@cam.ac.uk

\section{Data extraction}

Data were extracted on diagnostic criteria, presence or absence of antidepressant medication, method of randomisation and study design features, outcome measurement, blinding, and adverse effects.
Repetitive transcranial magnetic stimulation (rTMS) v placebo for major depressive disorder*

$\begin{array}{llll}\text { Outcome } & \begin{array}{l}\text { Weighted ABI } \\ (95 \% \mathrm{Cl})\end{array} & \text { RBI } & \text { (CI) } \\ \text { (CI) }\end{array}$

Improvement in depression rating scales $43 \%$ (25 to 61 ) $\quad 179 \%$ (104 to 254 ) 3 (2 to 4 )

${ }^{*}$ Follow up not reported. Abbreviations defined in glossary; RBI calculated from data in article.

\section{Main results}

5 trials were included ( 151 patients). All trials included patients who met DSM-III-R or DSM-IV criteria for a major depressive episode. In all 5 trials, placebo treatment consisted of placing the TMS coil at an ineffective angle of $45^{\circ}$ or $90^{\circ}$ to the scalp. All trials had blinded outcome assessments using the Hamilton Depression Rating Scale; 2 studies also used the Beck Questionnaire and 2 others also used the MontgomeryÅsberg Depression Rating Scale. More improvement in depression occurred in patients who received rTMS than in those who received placebo $(\mathrm{p}<0.001)$ (table). No patients withdrew from treatment because of adverse effects. 1 additional trial was identified of rTMS in the treatment of schizophrenia and showed no beneficial effects of rTMS

\section{Conclusions}

Meta-analysis of 5 randomised controlled trials of repetitive transcranial magnetic stimulation shows a beneficial effect in patients with major depression. Insufficient evidence exists to show an effect in other psychiatric disorders.

\section{COMMENTARY}

rTMS for the treatment of depressive illness has excited interest as a non-invasive and pain free alternative to electroconvulsive therapy (ECT). McNamara $e t$ al provide a distillation of the best evidence for the efficacy of rTMS in depression, and are justified in their conclusion that rTMS is effective. Since the end of the search period of the review (Jan 2000), 4 more randomised sham controlled trials have been published on the use of rTMS in depression, 3 of which show an advantage for rTMS..$^{1-3}$

Although a statistically significant decrease in depression scores is shown, rates of true remission are modest, which prompts caution in assessing the clinical utility of the treatment. The authors correctly point out that duration of effect and subgroup differential response have yet to be determined and call for longer term and larger studies. Furthermore, the optimal treatment parameters for rTMS need to be more precisely determined. Serious side effects of rTMS are extremely rare compared with ECT, at least in short term use, but the possibility of long term adverse effects on cognition and other areas cannot yet be ruled out. ${ }^{4}$ rTMS may find a specific place in the armamentarium of antidepressive treatments, but that place has not yet been defined. The extent, duration, and clinical role of its antidepressant effect, and its relation to ECT, have yet to be defined. The findings of this review will encourage further research.

Denzil R L Edwards, MBBS, MRCPsych

Pembury Hospital Tunbridge Wells, UK

1 Berman RM, Narasimhan M, Sanacora G, et al. A randomized clinical trial of repetitive transcranial magnetic stimulation in the treatment of major depression. Biol Psychiatry 2000;47:332-7.

2 George MS, Nahas Z, Molloy M, et al. A controlled trial of daily left prefrontal cortex TMS for treating depression. Biol Psychiatry 2000;48:962-70.

3 Garcia-Toro M, Mayol A, Arnillas H, et al. Modest adjunctive benefit with transcranial magnetic stimulation in medication-resistant depression. J Affect Disord 2001;64:271-5

4 Wassermann EM. Side effects of repetitive transcranial magnetic stimulation. Depress Anxiety 2000;12:124-9. 